\title{
Imposter Syndrome and the Peer-Review Crisis
}

Kirsten Winnie, MD I Jeremy T. Jackson, BA

PRIMER. 2021;5:46.

Published: 11/23/2021 | DOI: 10.22454/PRiMER.2021.584691

\section{To the Editor:}

We appreciate Dr Morley and Mr Grammer's thoughts on the importance of a healthy, equitable, and robust peer-review process, ${ }^{1}$ and share their concern that we have not yet attained this goal. We agree that this is critical to the success and growth of research in our discipline.

In our efforts to coach junior authors to submit to peer review for the first time, ${ }^{2}$ we received some interesting and unexpected feedback about peer review that is germane to this problem. Many participants in our coaching program were intimidated by the peer-review process, even from the perspective of a reviewer. They didn't really see it as something that all junior physicians can and should do; though they were motivated, they felt they lacked expertise. One participant noted, "people reviewing at journals would be more experienced...I might do it if I knew they were more like me." These participants had done research and were actively engaged in the peerreview process from the author perspective, yet they still felt underqualified to review. Impostor syndrome is a likely culprit for this hesitancy and could also partially account for the persistent inequality Dr Morley astutely points out.

At many levels, we need a concerted effort to coach and demystify peer review throughout the academic pipeline. For example, we could incorporate practicing peer review for students and residents as a new form of journal club. Learners could pair with faculty to serve as peer reviewers, even though they are still in training. Departments may consider academic incentive plans to motivate faculty to not only perform research ${ }^{3}$ but also to reward faculty taking the time to participate as reviewers.

Journals such as PRiMER and Family Medicine have made wonderful starts in making peer review more accessible, sharing pearls, and encouraging participation in editorials and at national meetings. ${ }^{4}$ We also appreciate these journals' practice of sharing the comprehensive review with all the reviewers' comments among the reviewers so that the reviewers themselves may benefit from the feedback and development. These practices are a great foundation, but we wonder if otherwise-inexperienced reviewers feel well equipped with these interventions. Journals could consider piloting programs to match experienced reviewers with inexperienced, but motivated, junior faculty to coach them through their first couple of peer reviews.

Any of these proposals to augment the pool of peer reviewers would represent an investment. Dr Morley and Mr Grammer have excellently outlined the benefits of such an investment: faculty development, healthy and rigorous scientific discourse, and service to the discipline. We absolutely need experienced and effective peer reviewers. A lack of time or motivation may not be the only barrier-increasing confidence and competence in the ability to peer-review is also critical.

\section{Acknowledgments}


Disclaimer: The views expressed within this publication represent those of the authors and do not reflect the official position of the Henry M. Jackson Foundation, the US Air Force, Uniformed Services University of the Health Sciences, or the US Government Department of Defense at large.

\section{Corresponding Author}

Jeremy T. Jackson, BA

Military Primary Care Research Network 4301 Jones Bridge Road, Bethesda, MD 20814. 301-295-0703.

jtjackson@hjf.org

\section{Author Affiliations}

Kirsten Winnie, MD - University of California, San Francisco, Fresno, CA

Jeremy T. Jackson, BA - Henry M. Jackson Foundation, Bethesda, MD | and Military Primary Care Research

Network, Uniformed Services University of the Health Sciences, Department of Family Medicine, Bethesda, MD

\section{References}

1. Morley CP, Grammer S. Now more than ever: reflections on the state and importance of peer review. PRiMER Peer-Rev Rep Med Educ Res. 2021;5:36. doi:10.22454/PRiMER.2021.216183

2. Winnie K, Jackson JT, Ledford CJ. Writing rounds: an innovation to increase physician scientific dissemination. PRiMER Peer-Rev Rep Med Educ Res. 2021;5:5. doi: 10.22454/PRiMER.2021.178789

3. Leverence R, Nuttall R, Palmer R, et al. Using organizational philosophy to create a self-sustaining compensation plan without harming academic missions. Acad Med. 2017;92(8):1133-1137. doi: 10.1097/ACM.0000000000001484

4. The Peer Review Process: Insight From the Editors of Family Medicine Journals. Presentation at 2019 STFM Annual Spring Conference. Toronto, Ontario, Canada; April 28, 2019.

Copyright $(2021$ by the Society of Teachers of Family Medicine 